\title{
Quimioterapia adjuvante para o tratamento do câncer de ovário e o cuidado
}

\section{farmacêutico: uma revisão integrativa}

Adjuvant chemotherapy for the treatment of ovarian cancer and pharmaceutical care: an integrative review

Quimioterapia adyuvante para el tratamiento del cáncer de ovario y la atención farmacéutica: una revisión integradora

\section{Resumo}

Este estudo teve como objetivo analisar os tratamentos quimioterápicos adjuvantes que vêm sendo adotados para o tratamento do câncer de ovário. Bem como, propor estratégias que podem ser adotadas para o cuidado farmacêutico a este paciente. Inicialmente, foi buscado os artigos na plataforma Capes, sendo incluídos artigos revisados por pares, publicados entre 2016 a 2021 e utilizado os seguintes termos: "pharmaceutical care protocol and câncer ovary and chemotherapy adjuvante". Foram encontrados 125 artigos e procedeu a leitura dos títulos e resumos, sendo que somente 10 artigos tiveram relação com o tema do trabalho e 7 trabalhos foram incluídos no estudo. Estes artigos, os pacientes faziam a quimioterapia convencional associado a bevacizumabe, indol-3-carbinol, epigalocatequina-3galato, pegfilgrastin e/ou filgrastin, vígil, lisado de tumor oxidado autólogo (OC-DC) e vacina de dez peptídeos (PEPDC), everolimo e letrozol. Os estudos demonstraram respectivamente: aumento da sobrevida livre de doença, porém é necessário mais estudos devido algumas controversas; ausência de toxicidade que exigisse alteração no regime de terapia padrão; que ao pacientes com maior pontuação de AIVD tem maior probabilidade de completar 4 ciclos de quimioterapia e menos probabilidade de apresentar toxicidade de grau 3 ou superior; melhora da resposta imune; a segurança, bem como alteração na sobrevida; uma taxa de PFS promissora, sendo que 47\% teve 12 semanas e toxicidade leve. Também, o desenvolvimento de formulação Nanopartículas de Paclitaxel-LDE (lipídeos não proteicos- PTX-LDE) levou uma melhora na resposta para o câncer de ovário. O papel do farmacêutico na equipe multiprofissional é muito importante, pois envolve o acompanhamento clínico do paciente, a prevenção de agravos a saúde da equipe devido a exposição aos quimioterápicos, a manipulação dos fármacos e detecção dos problemas relacionados aos medicamentos. Em síntese, ainda são escassos os estudos que visem a busca de alternativas terapêuticas mais eficazes para o tratamento do câncer de ovário.

Palavras-chave: Câncer de ovário; Quimioterapia adjuvante; Cuidado farmacêutico.

\begin{abstract}
This study aimed to analyze the adjuvant chemotherapy treatments that prove those adopted for the treatment of ovarian cancer. As well as the proportions that can be adopted for pharmaceutical care to this patient. Initially, articles were searched on the Capes platform, including peer-reviewed articles, published between 2016 to 2021 and using the following terms: "Pharmaceutical care protocol and cancer ovary and adjuvant chemotherapy". 125 articles were found and the titles and abstracts were read, with only 10 articles related to the work theme and 7 works were included in the study. In these articles, patients were undergoing conventional chemotherapy associated with bevacizumab, indole-3-carbinol, epigallocatechin-3-gallate, pegfilgrastine and/or filgrastine, vigil, autologous oxidized tumor lysate (OC-DC) and ten-peptide vaccine (PEP -DC), everolimus and letrozole. The studies showed, respectively: increased disease-free survival, but more studies are needed due to some controversial issues; Absence of toxicity that requires alteration of the standard therapy regimen; that patients with a higher IADL score are more likely to complete 4 cycles of chemotherapy and less likely to experience grade 3 or higher toxicity; improved immune response; safety as well as change in survival; a promising PFS rate, with $47 \%$ having 12 weeks and mild toxicity. Also, the development of basic Paclitaxel-LDE nanoparticles (non-protein lipids-PTX-LDE) has led to an improved response for ovarian cancer. The role of the pharmacist in the multidisciplinary team is very important, as it involves the clinical follow-up of the patient, the prevention of injuries and the health of the team due to exposure to
\end{abstract}


chemotherapy, drug manipulation and detection of drug-related problems. In summary, there are still few studies aimed at finding more effective therapeutic alternatives for the treatment of ovarian cancer.

Keywords: Ovary cancer; Adjuvant chemotherapy; Pharmaceutical care.

\begin{abstract}
Resumen
Este estudio tuvo como objetivo analizar los tratamientos de quimioterapia adyuvante que prueban los adoptados para el tratamiento del cáncer de ovario. Así como las proporciones que se pueden adoptar para la atención farmacéutica de este paciente. Inicialmente, se buscaron artículos en la plataforma Capes, incluidos artículos revisados por pares, publicados entre 2016 y 2021 y utilizando los siguientes términos: "Protocolo de atención farmacéutica y cáncer de ovario y quimioterapia adyuvante". Se encontraron 125 artículos y se leyeron los títulos y resúmenes, con solo 10 artículos relacionados con el tema del trabajo y se incluyeron 7 trabajos en el estudio. En estos artículos, los pacientes estaban sometidos a quimioterapia convencional asociada a bevacizumab, indol-3-carbinol, epigalocatequina-3-galato, pegfilgrastina y / o filgrastina, vigil, lisado tumoral oxidado autólogo (OC-DC) y vacuna de diez péptidos (PEP - DC), everolimus y letrozol. Los estudios mostraron, respectivamente: aumento de la supervivencia libre de enfermedad, pero se necesitan más estudios debido a algunos temas controvertidos; Ausencia de toxicidad que requiera alteración del régimen terapéutico estándar; que los pacientes con una puntuación IADL más alta tienen más probabilidades de completar 4 ciclos de quimioterapia y menos probabilidades de experimentar toxicidad de grado 3 o superior; respuesta inmune mejorada; seguridad, así como cambios en la supervivencia; una tasa de SLP prometedora, con un 47\% de 12 semanas y una toxicidad leve. Además, el desarrollo de nanopartículas básicas de paclitaxel-LDE (lípidos no proteicos-PTX-LDE) ha conducido a una mejor respuesta para el cáncer de ovario. El papel del farmacéutico en el equipo multidisciplinar es muy importante, ya que implica el seguimiento clínico del paciente, la prevención de lesiones y la salud del equipo por exposición a quimioterapia, manipulación de fármacos y detección de problemas relacionados con los fármacos. En resumen, aún existen pocos estudios encaminados a encontrar alternativas terapéuticas más efectivas para el tratamiento del cáncer de ovário.
\end{abstract}

Palabras clave: Cáncer de ovario; Quimioterapia adyuvante; Cuidado farmacêutico.

\title{
1. Introdução
}

O câncer de ovário é considerado um dos mais letais, pois frequentemente seu diagnóstico é tardio e mulheres. Estima-se que existam mais de 30 tipos diferentes de câncer de ovário, e há variação na incidência e perspectiva em termos dos diferentes tipos. No entanto, os números de óbitos pela doença devem aumentar em até 50\% nos próximos anos, tendo sido notificado 207.000 mortes e considerado o sétimo câncer mais comum em mulheres no mundo (World Ovarian Cancer Coalition, 2021).

A idade e susceptibilidade genética são considerados os $\mathrm{s}$ fatores de risco não modificáveis para o câncer de ovário, sendo que em $10 \%$ dos casos existe o componente genético ou familiar. Algumas mutações deletérias nos genes têm sido relacionadas a este câncer, tais como: BRCA1 no cromossomo 17q12-21 e BRCA2 no cromossomo 13q12-13 (Easton et al., 2995; Struewing, et al., 1997; Luiz, et al., 2008).

Dependendo do estadiamento e da diferenciação celular, o tratamento do câncer de ovário pode ter o objetivo curativo ou paliativo. O tratamento pode envolver procedimento cirúrgico de citorredução e quimioterápico neoadjuvante ou adjuvante. Em termos de quimioterapia, o tratamento de primeira deste tumor envolve 6 ciclos de carboplatina e paclitaxel (Lawrie, et al., 2015; Jiang, et al., 2017; Brasil, 2019), sendo a adição de outros fármacos, como bevacizumabe, uma importante ferramenta para o tratamento da doença em estágios avançados (Wright, et al., 2016; Rauh-Hain, et al., 2017).

Estudo avaliou a atividade e segurança do uso associado de paclitaxel e carboplatina (PCb) no tratamento neoadjuvante do câncer de mama, sendo avaliado a remissão completa da patologia (pCR) através da ausência de câncer invasivo. Dos 107 pacientes receberam tratamento semanal com PCb por 1 ano, apenas um foi diagnosticado com câncer de mama bilateral. Destes pacientes, $85,2 \%$ foram inicialmente diagnosticados com doenças em estágio III e a taxa de resposta clínica foi de $86,1 \%$ com taxa de remissão completa de 32,4\%. No caso de tratamento neoadjuvante, $19,4 \%$ dos pacientes alcançaram pCR. A incidência de neutropenia de grau 3-4 foi de 40,2\% e apenas um paciente foi relatado com neutropenia febril. Anemia grave e trombocitopenia ocorreram em 4,7\% e 0,9\%, respectivamente, dos pacientes. A neuropatia periférica foi frequente, mas nunca grave (Chen, et al., 2010). 
Esquemas terapêuticos alternativos têm sido utilizados no tratamento quimioterápico neoadjuvante e adjuvante do câncer de ovário. A quimioterapia neoadjuvante é considerada em situações clinicas em que o diagnóstico é realizado em estágios avançados e a citorredução máxima não puder ser realizada (Moura, et al., 2018). No caso de mulheres com a doença de alto risco (estádios IB e IC graus 2 e 3 e estádio IA grau 3) recomenda-se a utilização da quimioterapia adjuvante, pois metanálise demonstrou maior sobrevida em cinco e sobrevida livre de progressão do que aquelas que não receberam quimioterapia adjuvante (Adams, et al., 2010).

A presente revisão integrativa teve como objetivo analisar os tratamentos quimioterápicos adjuvantes que vêm sendo adotados para o tratamento do câncer de ovário. Bem como, propor estratégias que podem ser adotadas para o cuidado farmacêutico a este paciente.

\section{Metodologia}

Na construção da revisão integrativa foi utilizado o método comparativo, do tipo quali-quantitativo, sendo comparado os resultados dos diferentes estudos relacionados a quimioterapia adjuvante no câncer de ovário (Pereira, et al., 2018). O levantamento bibliográfico foi realizada no portal de periódicos Capes (Cesario et al., 2020; Cruz et al., 2020), adotando os descritores: pharmaceutical care protocol and ovary câncer and chemotherapy adjuvante.

Como critério de inclusão adotou-se: artigos revisados por pares; publicados nos últimos 5 anos; deveriam ser estudos primários; e abordar a quimioterapia adjuvante no tratamento do câncer de ovário. Foram excluídos artigos que abordassem a quimioterapia adjuvante de outros tipos de câncer; relatos de estudos in vitro e em animais; artigos de revisão e sem relação ao tema. Também se excluiu artigos que não fossem redigidos em português, inglês e espanhol.

Foram encontrados na busca inicial 155 artigos no total, destes apenas 125 foram revisados por pares. Na primeira etapa, foram lidos os títulos e resumos, sendo excluídos os artigos que não cumpriram os critérios de inclusão, sendo a principal causa de exclusão o tratamento adjuvante de outros tipos de tumores, restando apenas 10 artigos que foram lidos na íntegra e excluídos 3 artigos por utilizarem estudos in vitro e/ou em animais. Na segunda etapa, os 7 artigos incluidos foram lidos na integra e coletadas as seguintes informações: objetivo, estadiamento, esquema terapêutico adotado e síntese dos resultados. O fluxograma faz a síntese do protocolo adotado neste trabalho (Figura 1).

Figura 1: Fluxograma da seleção dos artigos.

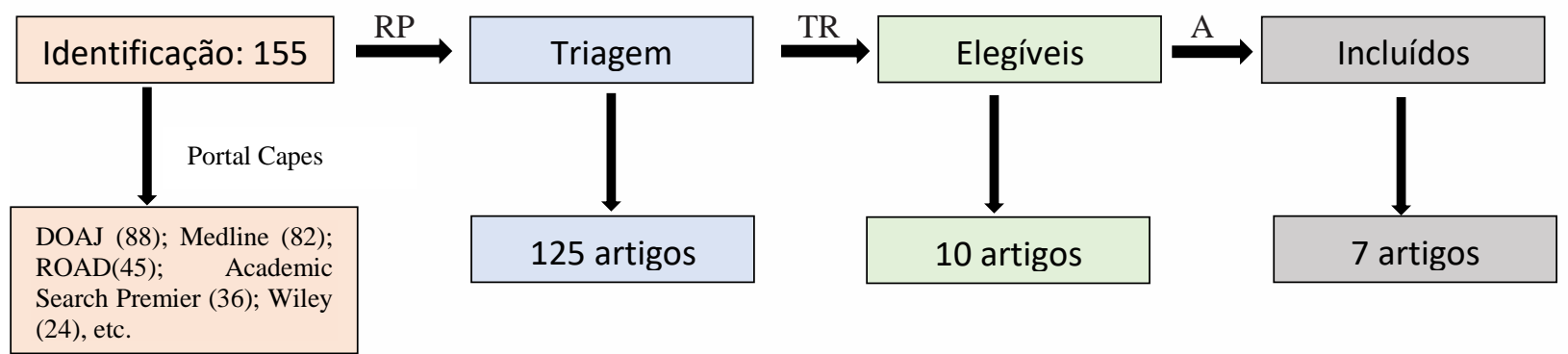

Legenda: RP: Revisados por pares: TR: leitura dos títulos e resumos; A: leitura do artigo na integra. Fonte: Autores (2021).

\section{Resultados e Discussão}

$\mathrm{Na}$ presente revisão integrativa foram incluídos estudos que avaliaram a toxicidade da quimioterapia (Grazini, et al., 2017), a eficácia (Kiseley, et al., 2018), prolongamento do tempo de sobrevida da paciente com câncer de ovário (del Carmen, et al., 2016; Oh, et al., 2016; Colon-Otelo, et al., 2017) e segurança. Ainda, um estudo avaliou o tratamento com quimioterápicos em portadoras de câncer de ovário e com idade avançada (von Gruenigen, et al., 2017). Por fim, incluiu um 
artigo que, teoricamente não tinha relação direta com a quimioterapia adjuvante, porém avaliou um aspecto muito importante que é aumento da sobrevida, tendo sido utilizado como estratégia terapêutica a vacina (Tabela 1) (Sarivalasis, et al., 2019).

No câncer de ovário, em geral, o diagnóstico é tardio e a taxa de letalidade é elevada (Sopik, et al., 2015), logo a quimioterapia adjuvante tem como objetivo o prolongamento da sobrevida da paciente (Moura, et al., 2018). Além do tratamento convencional, tendo sido proposto a inclusão de anticorpo monoclonal, bevacizumabe, para ampliar o tempo de sobrevida livre da progressão. A inclusão de bevacizumabe ainda é controversa, pois em estudos randomizados foram obtidos resultados controversos. Em um, onde foram incluídas pacientes em estadiamento de III a IV houve um aumento na sobrevida livre da progressão (Komiyama, et al., 2018), enquanto em outro estudo não foi possível detectar esta diferença na sobrevida (Chan, et al., 2016).

O uso associado da carboplatina ao paclitaxel tem sido relacionado a reações adversas hematológicas (anemia, trombocitopenia, neutropenia e neutropenia febril), alopecia, ototoxicidade e neuropatia periférica (du Bois, et al., 2010). Muitas vezes, o desenvolvimento de nova formulação pode contribuir para redução da toxicidade do medicamento (Graziani, et al., 2017). Também, estudar novos esquemas terapêuticos é importante (Colon-Otero, et al., 2017). 
Research, Society and Development, v. 10, n. 16, e497101620638, 2021

(CC BY 4.0) | ISSN 2525-3409 | DOI: http://dx.doi.org/10.33448/rsd-v10i16.20638

Tabela 1: Artigos incluídos e seus objetivos.

Referências

Objetivos dos artigos

1. Graziani, S.R., et al. Phase II study of paclitaxel associated with lipid core nanoparticles (LDE) as third-

line treatment of patients with epithelial ovarian carcinoma. Med Oncol., v.34, p.151, 2017

2. Kiselev, V.I. et al. A new promising way of maintenance therapy in advanced ovarian cancer: a

comparative clinical study. BMC Cancer, v.18, p.904, 2018

O estudo de fase II avaliou a toxicidade paclitaxel associada com nanopartículas com núcleo lipídico (LDE) em pacientes com câncer de ovário

3. von Gruenigen, V.E., et al. Chemotherapy completion in elderly women with ovarian, primary peritoneal or fallopian tube cancer - An NRG oncology/Gynecologic Oncology Group study. Gynecologic Oncology, v.144, p.459-467, 2017

e epigalocatequina-3-galato (EGCG) conduzido antes, durante e após o tratamento combinado em comparação com o tratamento combinado sozinho no câncer de ovário avançado.

Explorar a associação entre as Atividades Instrumentais de Vida Diária pré-tratamento (AIVD) e a capacidade do paciente de completar 4 ciclos de quimioterapia sem redução da dose ou atraso no tratamento de N7 dias para pacientes com 70 anos e mais velhos com câncer primário de ovário, peritoneal ou trompa de Falópio. Os objetivos secundários incluíram explorar se idade, medidas geriátricas e resultados relacionados ao paciente (PROs) foram correlacionados com toxicidade ou conclusão da quimioterapia.

4. Oh, J., et al. Phase II study of Vigil@ DNA engineered immunotherapy as maintenance in advanced stage ovarian câncer. Gynecologic Oncology, v.143, p. 504-510, 2016.

Demonstrar o prolongamento da Sobrevida Livre de Recorrência com manutenção de Vigil em pacientes com câncer de ovário nos estágios III / IV atingindo completa resposta clínica (cCR) seguir a gestão da linha de frente; portanto, com doença residual mínima (MRD).

5. Sarivalasis, A., et al. A Phase I/II trial comparing autologous dendritic cell vaccine pulsed either with personalized peptides (PEP-DC) or with tumor lysate (OC-DC) in patients with advanced high-grade ovarian serous carcinoma. J Transl Med., v.17, p.391, 2019

6. del Carmen, M. G., et al. Phase I/II Study of Carboplatin and Pralatrexate in Patients with Recurrent Platinum Sensitive Ovarian, Fallopian Tube or Primary Peritoneal Cancer. Cancer, v. 122, n.21, p.32973306,2016

7. Colon-Otero, G. et al. Phase 2 trial of everolimus and letrozole in relapsed estrogen

7. Colon-Otero, G. et al. Phase 2 trial of everolimus and letrozole in relapsed estrogen
receptor-positive high-grade ovarian cancers. Gynecologic Oncology, v.146, p. 64-68, 2017 O objetivo do estudo randomizado de fase I / II em pacientes com câncer de ovário avançado é comparar a imunogenicidade e avaliar a segurança e viabilidade de duas vacinas personalizadas de células dendríticas (DC).

Relatar a dose máxima tolerada de pralatrexato associada a carboplatina e a taxa de resposta. Os objetivos secundários incluíram a medição de sobrevida livre de progressão e sobrevida global, avaliação das toxicidades do tratamento e caracterização de PK deste regime.

Avaliar o potencial benefício clínico da combinação de Inibidores de Aromatase e everolimus em ERpositivo câncer de ovário em um ensaio clínico de fase 2 com o objetivo primário taxa de Sobrevida Livre de Progressão (PFS) de 12 semanas.

\section{Fonte: Autores}


Estudo demonstrou a presença de receptores de LDL nas células tumorais de ovário, estes receptores estão evolvidos na captação de nanopartículas núcleo lipídico não proteico (LDE). Além disso, receptores LRP-1 também estão presentes nas células tumorais e têm a capacidade de internalizar LDL nativo (Strickland et al., 1995; Herz \& Strickland, 2001) e paclitaxel (PTX) transportado em núcleo de lipídeo não proteico nanopartículas (LDE; PTX-LDE). Logo, o desenvolvimento da formulação pode contribuir para ampliar a captação do fármaco pelo tumor (Graziani, et al., 2017).

A toxicidade dos quimioterápicos utilizados no tratamento do câncer de ovário tem sido relatada, sendo importante a busca de medicamentos com menor potencial tóxico. Em relação à toxicidade PTX-LDE (dose usual= $175 \mathrm{mg} / \mathrm{m}^{2}$, aos 14 pacientes, totalizando 74 ciclos) não foram observadas alterações clinicas significativas e sugestivas de efeitos tóxicos, também não houve alterações laboratoriais (Tabela 2) (Graziani, et al., 2017). As toxicidades das formulações PTX padrão, nas quais o óleo de rícino polioxietilado é usado como veículo, consistem principalmente em mielotoxicidade, especialmente neutropenia (Maier et al., 1997), bem como mucosite (Raber-Durlacher, et al., 2000), arritmias ventriculares, bradicardia e bloqueios de condução (Arbuck, et al., 1993), reações de hipersensibilidade, como dispneia, urticária e a hipotensão arterial estão relacionada ao veículo e não à PTX (Weiss, et al., 1990).

A ausência de toxicidade clínica e laboratorial aumenta a possibilidade da utilização de doses mais elevadas PTX veiculada na LDE com o objetivo de aumentar a PFS, bem como elevar a tolerabilidade ao tratamento. Outro estudo utilizou o mesmo medicamento para o tratamento do câncer de mama avançado, na dose de $250 \mathrm{mg} / \mathrm{m}^{2} / 3 \mathrm{em} 3$ semanas e não se observou efeitos tóxicos (Graziani, et al., 2017).

Outro estudo avaliou a eficácia da associação de indol-3-carbinol (I3C) e/ou epigalocatequina-3-galato (EGCG) carboplatina/paclitaxel (quimioterapia neoadjuvante, citorredução e adjuvante), sendo que estas associações contribuíram com a elevação da sobrevida dos pacientes. Estas associações ainda contribuíram para a melhoria da qualidade de vida e da capacidade funcional dos pacientes. A administração de I3C e EGCG não afetou negativamente a condição geral dos pacientes e não causar quaisquer eventos adversos adicionais (AEs) além do causados pela administração de drogas quimioterápicas padrão. Não houve AEs exigindo redução no padrão dosagens de quimioterapia ou quaisquer alterações no regime de terapia padrão e / ou de manutenção. Não houve casos de descontinuação, ou alteração do recomendado dosagens de fármacos contendo I3C e EGCG e nenhuma morte relacionada ao tratamento (Tabela 2) (Kiselev, et al., 2018).

Os produtos naturais I3C e EGCG suprimem a proliferação de células tumorais, induzindo seletivamente a parada do ciclo celular e a apoptose. Estudo demonstrou a atividade antiangiogênica, antimigratória, antimetastática, antiinflamatória e antioxidante. I3C e o metabolito 3,3'-diindolylmetano (DIM) ligam aos receptores de hidrocarbonetos de arila, que tem sido relacionado ao desenvolvimento do câncer. Estes compostos ainda alteram o metabolismo de xenobiótico e estrogênio, melhorando a razão 2-hidroxestrona / 16 $\alpha$-hidroxestrona no sangue e tecidos dependentes de estrogênio. I3C, DIM e EGCG também têm atividade antitumoral epigenética inibindo as principais enzimas como DNA metiltransferase (Fang, et al., 2003; Haefele, et al., 2007; Lyn-Cook, et al., 2010; Wu, et al., 2013) e histona desacetilase (Li et al., 2010; Pandey et al., 2010; Beaver, et al., 2012). O EGCG é considerado promissor, com atividade epigenética de desmetilação de DNA comparável aos atuais medicamentos aprovados pela FDA (Li \& Tollefsbol, 2010). I3C, DIM e EGCG podem modular perfis de expressão de miRNA não codificantes, levando a inibição do crescimento de células tumorais, indução de apoptose, reversão da transição epitelial-mesenquimal, ou aumento da eficácia de terapias convencionais contra o câncer (Li, et al., 2010).

Outro estudo avaliou o tratamento do câncer de ovário com apenas a terapia padrão (Carboplatina e praclitaxel) e associada Pegfilgrastin (CP) ou a Filgrastin (G-CFS) em pacientes geriátricas (Tabela 2) (von Gruenigen, et al., 2017). A maioria dos estudos de tratamentos medicamentosos não incluem pacientes idosas, que podem apresentar resposta ao tratamento diferente a do adulto. Logo, estudos clínicos envolvendo idosas é muito importante para avaliar a resposta e 
possíveis eventos adversos (Hurria, et al., 2011; Dale, et al., 2012; Hurria et al., 2013; Hurria, et al., 2014; Hurria, et al., 2015; von Gruenigen, et al., 2017).

A avaliação do tratamento quimioterápicos na geriátrica deve incluir uma avaliação do estado funcional, condições comorbidades presentes nesta etapa da vida, estado psicológico, suporte social, estado nutricional e cognição (Wildiers, et al., 2014; von Gruenigen, et al., 2017). Na avaliação do estado funcional precisa inclui a capacidade do paciente de realizar atividades instrumentais de vida diária (AIVD), que são habilidades de autocuidado que permitem o funcionamento independente dentro da comunidade e atividades de vida diária (AVD) e habilidades básicas de autocuidado, como a capacidade de tomar banho e se vestir. Para tais avaliações podem ser usadas o questionário PRO, onde as respostas são coletadas diretamente do paciente (Deshpande, et al., 2011; von Gruenigen, et al., 2017), sendo considerados os sintomas apresentados, estado funcional e a qualidade de vida (QV) (von Gruenigen, et al., 2017).

No estudo com mulheres com mais 70 anos, as pacientes no grupo CP, tratadas com carboplatina + Paclitaxel + Pegfilgrastin, apresentaram escores basais de AIVD mais altos. A pontuação de AIVD de base parece não estar associada com conclusão de 4 ciclos de quimioterapia sem redução da dose ou atrasos, porém teve relação com a redução da toxicidade. Após o ajuste para o regime de quimioterapia, AIVD também foi associado à sobrevida global para pacientes que receberam CP. Em síntese, os pacientes com maior pontuação ide AIVD tem maior probabilidade de completar 4 ciclos de quimioterapia e menos probabilidade de apresentar toxicidade de grau 3 ou superior (Tabela 2) (von Gruenigen, et al., 2017).

A Pegfilgrastin é um fator de crescimento de leucócito, aprovado pelo FDA para diminuir a incidência de infecção em pacientes com neutropenia febril e com doenças malignas não mieloides em tratamento com mielossupressores. Também, este medicamento é indicado para aumentar a sobrevida em pacientes expostos agudamente a mielossupressores e radiação. Estudo randomizado, duplo-cego, de fase 3 comparou uma única dose fixa de $6 \mathrm{mg}$ de Pegfilgrastin, administrada uma vez por ciclo de quimioterapia com injeções diárias de Filgrastin, sendo a pegfilgratin tão eficaz quanto diariamente Fllgrastim para o tratamento da neutropenia em pacientes de alto rico (Green, 2011).

Tabela 2: Estadiamento do câncer, tratamento utilizado e síntese dos resultados.

\begin{tabular}{|c|c|c|c|}
\hline $\mathrm{n}^{\mathrm{o}}$ & $\begin{array}{l}\text { Estadiamento dos } \\
\text { pacientes incluídos }\end{array}$ & Tratamento & Síntese dos resultados \\
\hline 1 & CEORDP & $\begin{array}{l}\text { Grupo 1:Nanopartículas de Paclitaxel-LDE } \\
\text { (lipídeos não proteicos- PTX-LDE) } \\
\text { Monitorados com: exames de imagens e } \\
\text { bioquímicos }\end{array}$ & $\begin{array}{l}\text { Grupo 1: } 74 \text { ciclos sem toxicidade; sobrevida livre de } \\
\text { progressão média (PFS)=3,0 meses. E; } 4 \text { a PFS foi de } 6 \\
\text { meses; em } 2 \text { a FPS } 1 \text { ano; menos } 4 \text { entre } 14 \text { pacientes } \\
\text { tiveram parada do tumor pelo tratamento e benefício. }\end{array}$ \\
\hline 2 & $\begin{array}{l}\text { Estágio III a IV Câncer } \\
\text { de ovário }\end{array}$ & $\begin{array}{l}\text { Grupo 1: } 200 \mathrm{mg} \text { de indol-3-carbinol (I3C) } \\
\text { (VO, 2x/dia), por } 5 \text { anos+ carboplatina- } \\
\text { paclitaxel (Ciclos de } 2 \text { a } 3 \text { semanas)- } \\
\text { quimioterapia neoadjuvante, citorredução e } \\
\text { adjuvante } \\
\text { Grupo } 2: 200 \mathrm{mg} \text { de } \mathrm{I} 3 \mathrm{C} \text { (VO, } 2 \mathrm{x} \text { ao dia) }+200 \\
\mathrm{mg} \text { de epigalocatequina-3-galato (EGCG) } \\
\text { (VO/2x/dia), por } 5 \text { anos+ carboplatina- } \\
\text { paclitaxel (Ciclos de } 2 \text { a } 3 \text { semanas)- }\end{array}$ & $\begin{array}{l}\text { PFS: grupo } 1=42,5 \text { meses; grupos } 2=48,5 \text {; grupo } 3=34,5 \\
\text { meses; grupo } 4=22 \text { meses; grupo } 5=22 \text { meses } \\
\text { Sobrevida geral OS: grupo } 1=60 \text { meses; grupos } 2 \text { e } 3=46 \\
\text { meses; grupo } 4=44 \text { meses; grupo } 5=39 \text { meses } \\
\text { Associação carboplatina-paclitaxel (Ciclos de } 2 \text { a } 3 \\
\text { semanas)- quimioterapia neoadjuvante, citorredução e } \\
\text { adjuvante associada ao I3C por longo prazo de } \\
\text { adeministração parece ser promissora }\end{array}$ \\
\hline
\end{tabular}
quimioterapia neoadjuvante, citorredução adjuvante;

Grupo 3: $200 \mathrm{mg}$ de I3C + $200 \mathrm{mg}$ de EGCG $(\mathrm{VO}, 2 \mathrm{x}$ ao dia) + carboplatina-paclitaxel (quimioterapia neoadjuvante, citorredução e adjuvante de longo prazo (ciclos 2 a 3 meses) Grupo 4: carboplatina-paclitaxel (Ciclos de 2 a 3 semanas)- citorredução e quimioterapia adjuvante

Grupos 5: carboplatina-paclitaxel (Ciclos de 2 a 3 semanas)- quimioterapia neoadjuvante, citorredução e adjuvante 
$3 \geq 70$ anos $\mathrm{CEO}$ estágio I-IV, peritônio ou trompa de Falópio.

4 Estágios III / IV CEO, câncer de falópio ou câncer peritoneal primário

5 Câncer de ovário seroso de alto grau (HGSOC) estágio III ou IV

6 Câncer recorrente de ovário, trompa de Falópio ou peritoneal

7 Recidiva mensurável de ovário ER, trompa de Falópio ou carcinomas peritoneais primários
Grupo CP: carboplatina + Paclitaxel + Pegfilgrastin (por 3 semanas)

Grupo G-CSF: Carboplatina + Paclitaxel + Filgrastin (por 3 semanas)

Grupo C: Carboplatina (ciclos de 3 samanas) Neoadjucantes (4 ciclos)+ citorredução cirúrgica (para pacientes que não tiveram cirurgia) e / ou adjuvante

Grupo 1 (31 pacientes): citorredução cirúrgica e adjuvante carboplatina / paclitaxel ou neoadjuvante+ Vigil

$\left(1.0 \times 10^{7}\right.$ células/Intradérmica /mês por 4 e 12 doses

Monitorado (mensal): função hematológica, enzimas hepáticas, função renal e eletrólitos, função imune em série, incluindo ELISPOT, função da célula mononuclear e células tumorais (início, antes da injeção Vigil e nos meses 2, 4, 6 e EOT).

Grupo 2 (11 pacientes): citorredução cirúrgica e adjuvante carboplatina / paclitaxel ou neoadjuvante

Citorredução + quimioterapia

Grupo 1: lisado de tumor oxidado autólogo (OC-DC)

Grupo 2: OC-DC +vacina dez peptídeos (PEPDC)

Fase I:dose fixa de carboplatina (i.v.) + dose crescente de pralatrexato (i.v.), ao longo de 3060 minutos a cada 28 dias). Dose inicial de pralatrexato $=30 \mathrm{mg} / \mathrm{m}^{2}$, nos dias 1 e 15 , escalonado por $15 \mathrm{mg} / \mathrm{m}^{2}$ no ciclo completo Dose máxima administrada ou a toxicidade limitante da dose (DLT) foi avaliada.

Fase II: foi definida como um nível de dose sob DLT e anotada como o máximo dose tolerada (MTD). As toxicidades foram classificadas usando as escalas National Cancer Institute (NCI) Common Terminology Criteria for Advse Eventos versão 4.0

Grupo intervenção: everolimo $(10 \mathrm{mg}$ $\mathrm{VO} /$ dia $)+$ letrozol $(2,5 \mathrm{mg}, \mathrm{VO} / \mathrm{dia})$. Ocorrência da doença progressão ou desenvolvimento de toxicidade: redução da dose do everolimo para $5 \mathrm{mg}$ ou $2,5 \mathrm{mg}$ em caso de toxicidade 3 ou grave
$\mathrm{CP}=92 \%$ completaram 4 ciclos, mais reações adversas (neutropenia, anemia, diarreia e desidratação); 4 óbitos (1 problema cardíaco e 1 aspiração); escores basais de atividades do dia-a-dia (AIVD) mais altos; redução da dose foi associado a conclusão de 4 ciclos e razão de chances (OR) de toxicidade grau 3+

$\mathrm{C}=75 \%$ completaram 4 ciclos; anemia e trombocitopenia foram relatadas; 3 óbitos devido a progressão da doença;

Grupo 1: nenhuma toxicidade $\geq$ Grau 3 relacionada ao produto foi observada. Indução de células $\mathrm{T}$ ativadas contra tumor autólogo individual pré-processado Vigil em comparação com o pré-Vigil linha de base usando resposta ELISPOT de IFN $\gamma$ (30/31 ELISPOT negativo pré-vigília a 31/31 ELISPOT positivo pós-vigília, mediana 134 pontos). $\mathrm{RFS}=$ média de 826 dias / mediana de 604 dias no braço Vigil

Grupo 2: RFS= média de 481 dias / mediana de 377 dias

Pré-tratamento com OC-DC seguida vacinação PEP-DC parece induzir a propagação do epítopo, a resposta imune contra ampla gama de antígenos específicos do paciente e para melhorar clones pré-existentes de células $\mathrm{T}$ específicas do NeoAg.

Fase I: 25/ 30 pacientes apresentaram eventos adversos; 4 pacientes foram de grau 3 e 2 tiveram neutropenia febril e reação de hipersensibilidade

Fase II: $15 / 20$ pacientes apresentaram eventos adversos; 1 paciente trombocitopenia grau 3; 1 neutropenia febril; 1 reação de hipersensibilidade. A redução da dose, reduziu a neutropenia.

Grupo intervenção: PFS 47\% mediano de 3,9 meses, PFS de 6 meses de 32\%; 3 pacientes estavam vivos em 18,6 meses; $3(16 \%)$

resposta parcial confirmada; nenhum teve resposta completa; 7s (37\%) estabilização da doença com controle de 53\%; 4 (21\%) redução da doença em 50\%; 4resposta parcial à terapia; 2 tiveram uma melhor resposta de doença estável; 12 pacientes (63\%):pelo menos 1 toxicidade grau 3 ou piora do evento adverso (AE); 3 pacientes (16\%) grau 4 ou piora de $\mathrm{AE} ; 3$ pacientes diminuição de neutrófilos de grau 4, 1 anemia de grau 4 e diminuição de leucócitos de grau 4, 1de grau 5 não relacionado ao tratamento. AEs de grau 3 (10\% dos pacientes):dor abdominal (16\%), intestino delgado obstrução (16\%), mucosite oral (11\%) e anemia (11\%).

Fonte: Autores.

Estudo preliminar em pacientes com câncer ovariano tem demonstrado que este é imunogênico e são apropriados para abordagens imunomediadas (Kandalaft, et al., 2011), como pelo Vigil. Aumentos significativos em TGF $\beta 1$ foram observados em ambos os tecidos de câncer de ovário primário e recorrente (Bristow, et al., 1999) e a presença de proteínas imunossupressoras intrínsecas, como TGF $\beta$, pode trazer benefícios clínicos. Vigil® Ovarian (gemogenovatucel- T), incorporando o cDNA rhGMCSF (fator de estimulação de colônia de granulócitos-macrófagos humanos recombinantes) e o 
shRNA bifuncional (ácido ribonucléico em grampo curto; uma nova fração de interferência de RNA) visando furina (para bloquear furina conversão mediada de pró-proteínas TGF $\beta$ em TGF $\beta 1$ e $\beta 2$ ativos, imunossupressores). Além disso, usando tecido tumoral autólogo, Vigil (vacina) fornece a matriz completa do antígeno específico do tumor dentro do células do próprio paciente, e estudo de Fase I em pacientes com tumor sólido, incluindo pacientes com câncer de ovário demonstrou segurança marcante da imunoterapia Vigil (Senzer, et al., 2012). Acompanhamento de longo prazo, em pacientes tratados com esta vacina, demonstrou a segurança, bem como alteração na sobrevida (Senzer, et al., 2013).

Estudo realizado em pacientes com câncer de ovário em quimioterapia convencional e receberam Vigil demonstrou prolongamento de sobrevida livre de recorrência (RFS) e com doença residual mínima (MRD). Em conjunto com a segurança demonstrada, a alta taxa de indução de ativação de células T e a correlação com a melhora no RFS justifica uma avaliação adicional de Fase II / III do Vigil (Tabela 2) (Oh, et al., 2016).

Outro estudo avaliou a contribuição da vacinação para gerar uma nova resposta antitumoral e amplificar uma resposta imune existente. Estudo demonstrou a importância do uso do lisado de tumor oxidado autólogo (OC-DC), seguida da vacina dez peptídeos (PEP-DC) (Tabela 2). Vacinas contra o câncer baseadas em NeoAgs têm a vantagem de ser mais específico para o tumor, reduzindo o potencial de tolerância imunológica e induzindo imunogenicidade robusta (Sarivalasis, et al., 2019). Peptídeos de neoantígeno (NeoAg) exibidos na superfície da célula tumoral por moléculas de antígeno de leucócito humano mostram especificidade tumoral requintada e podem induzir rejeição tumoral mediada por células T (Li, et al., 2021).

Quando as vacinas têm como alvo autoantígenos não mutados definidos ou compartilhados antígenos que são superexpressos no tumor, a eficácia é frequentemente baixa porque a reatividade das células $\mathrm{T}$ a autoantígenos é reduzido devido à tolerância central (Schietinger et al., 2008). Alternativamente, neoantígenos (NeoAgs), que surgem de somáticos, são alvos eficazes para a infiltração de tumores e podem apresentar maior eficácia contra tumores com carga de mutação moderada ou alta. Estudos de fase I usando NeoAg personalizado relataram imunogenicidade e resultados clínicos interessantes de segurança e eficácia (Carreno, et al., 2015; Ott, et al., 2017; Sabin, et al., 2017; Ott, et al., 2018).

Estudo de Fase I / II avaliou o impacto da redução da dose do pralatrexato na eficácia e tolerabilidade da terapia combinada de carboplatina e pralatrexato, no manejo de pacientes com sensibilidade à platina câncer e com ovariano epitelial recorrente, trompa de Falópio ou peritoneal. A maioria dos pacientes apresentaram eventos adversos em níveis aceitáveis, tanto nos estudos de fase 1 como de fase 2 (Tabela 2). Este estudo tempo relacionar os eventos adversos aos aspectos farmacocinéticos ocasionados pela redução da dose, porém não foram observadas alterações significativas. Logo, é importante identificar os fatores que podem predispor ao paciente aos efeitos tóxicos desta associação (del Carmen, et al., 2016).

A combinação de everolimo e letrozol está associada a uma taxa de PFS promissora, sendo que 47\% teve 12 semanas. Toxicidade leve relacionada ao medicamento foi observada consistindo principalmente de anemia e mucosite oral. Esta combinação merece avaliação adicional em recidiva de câncer de ovário receptor de estrogênio (ER) positivo (Tabela 2) (Colon-Otero, et al., 2017).

O letrazol é um inibidor da aromatase, sendo a terapia com inibidor de aromatase de agente único (AI) associada à atividade clínica limitada no câncer de ovário, isto é, alguns ensaios clínicos de fase 2 demonstraram taxas de resposta variando de 0\% a 38\% no cenário de recaída (Bowman, et al., 2002; del Carmen, et al., 2003; Papadimitriou, et al., 2004; Krasner, et al., 2005; Gourley, et al., 2006; Tchekmedyian, et al., 2006; Kavanagh, et al., 2007; Li, et al., 2008; Colon-Otero, et al., 2017). No maior ensaio de fase 2 , a terapia com letrozol no câncer de ovário recidivante foi associada a taxa de sobrevivência livre de progressão (PFS) em 12 semanas de 20\% (Bowman, et al., 2002). Um potencial mecanismo de resistência está relacionado a interferência da sinalização ER (Osborne, et al., 2006), levando ativação de várias vias de sinalização, incluindo o PI3K / AKT /via mTOR, que é inibida pelo everolimo (Simoncini \&Hafezi-Moghadam, 2000; ColonOtero, et al., 2017). 


\subsection{Cuidado farmacêutico ao paciente em quimioterapia para o câncer de ovário}

Ao se analisar os 7 artigos fica claro que, a maioria dos estudos visavam a incorporação de novo tratamento visando melhorar a eficácia do tratamento e não impactar na toxicidade. Alguns trabalhos sugeriram a incorporação de vacinas (Oh, et al., 2016; Sarivalasis, et al., 2019) e produto que atua na resposta imune. Muitas vezes, estes produtos possuem preço elevado e deve ser avaliado o benefício de sua incorporação e o farmacêutico pode participar ativamente nestes estudos que visem a avaliação de novas tecnologias de saúde. A avaliação de tecnologia em saúde envolve uma avaliação das consequências da inserção do produto em curto e longo prazo, sendo considerado as questões clínicas, sociais, econômicas éticas e organizacionais relacionadas ao uso da tecnologia em saúde. Esta pesquisa deve adotar métodos para a tomada de decisão, e deve ser feita de forma imparcial, transparente e sistemática (Brasil, 2016).

Dentro da equipe multidisciplinar envolvida no tratamento do câncer, o farmacêutico é responsável por toda a logística do medicamento (seleção, programação, aquisição, armazenamento e dispensação), bem como pela a manipulação dos fármacos. As metas globais relacionadas à assistência farmacêutica envolvem desde a promoção do cuidado de alta qualidade com a melhoria dos resultados, bem como a proteção dos trabalhadores quanto aos riscos aos fármacos, prevenção dos erros relacionados aos medicamentos e gerenciamento dos medicamentos (Silva \& Osório-de-Castro, 2019).

De acordo com o Consenso de Granada, realizado pelo Comitê de Consenso (2002), alguns problemas envolvem a utilização dos medicamentos (PRM), tais como: necessitude, isto é, o paciente realmente tem o problema de saúde (PRM1), ou este problema é consequência do uso de medicamento que não necessita (PRM2); ausência de resposta, sendo que problema pode estar relacionado a falta de resposta ao tratamento, devido a não adesão ao tratamento, interações farmacocinéticas e limitações de acesso aos medicamentos; por fim a segurança do paciente (PRM 4), envolvendo as consequência do uso de medicamento, como as reações adversas aos medicamentos, tóxicas e interações medicamentosas contraindicadas e graves, podendo ocorrer danos à saúde. Além disso, o paciente pode sofrer problemas de saúde resultante de uma incerteza quantitativa (PRM5) ou resultante de uma insegurança qualitativa.

$\mathrm{Na}$ presente revisão integrativa, observou-se a preocupação, em especial, com os eventos adversos. O farmacêutico, dentro da equipe multiprofissional, pode ser o responsável pela a detecção destes eventos, bem como participar de ações que visem a redução destes problemas. Por fim, o paciente oncológico pode ser polimedicado, aumentando o risco de reações adversas e cabe ao farmacêutico a análise destas interações e sua classificação relacionada ao risco clínico.

\section{Conclusão}

Esta revisão integrativa permitiu estabelecer alguns problemas relacionados ao tratamento de primeira escolha para o câncer de ovário e possíveis estratégias para aumentar a eficácia terapêutica sem impactar na toxicidade. As vacinas para o tratamento do câncer de ovário parecem ser promissoras, bem como os antagonistas de ER e inibidores da aromatase. Também, associação da quimioterapia ao I3C se mostrou bastante promissora. Conforme pode se observar, ainda existe uma escassez de estudos que visem a melhoria da quimioterapia adjuvante, havendo necessidade de estudos adicionais.

O papel do farmacêutico na equipe multiprofissional envolve a promoção do cuidado de alta qualidade com a melhoria dos resultados, a proteção dos trabalhadores quanto aos riscos aos fármacos, prevenção dos erros relacionados aos medicamentos e gerenciamento dos medicamentos (Silva \& Osório-de-Castro, 2019).

\section{Referências}

Adams G, Zekri J, Wong H, Walking J, \& Green JA. (2010). Platinum-based adjuvant chemotherapy for early-stage epithelial ovarian cancer: single or combination chemotherapy? BJOG., 117(12), 1459-67. 
Adams, G., Zekri, J., Wong, H., Walking, J. \& Green, J.A. (2010). Platinum-based adjuvant chemotherapy for early-stage epithelial ovarian cancer: single or combination chemotherapy? BJOG., 117(12),1459-67.

Arbuck, S. G, Strauss, H., Rowinsky, E., Christian, M., Suffness, M., Adams, J., Oakes, M., McGuire, W., Reed, E., \& Gibbs, H. (1993). A reassessment of cardiac toxicity associated with Taxol. J Natl CancerInst Monogr., 15:117-30.

Beaver, L. M., Yu, T. W., Sokolowski, E. I., Williams, D. E., Dashwood, R. H., \& Ho, E. (2012). 3,3'- Diindolylmethane, but not indole-3-carbinol, inhibits histone deacetylase activity in prostate cancer cells. Toxicol Appl Pharmacol., 263(3), 345-51.

Bowman, A., Gabra, H., Langdon, S. P, Lessells, A., Stewart, A., Young, A., \& Smyth, J. F. (2002). CA125 response is associated with estrogen receptor expression in a phase II trial of letrozole in ovarian cancer: identification of an endocrine-sensitive subgroup. Clin. Cancer Res. 8, 2233-2239.

Brasil, Ministério da Saúde. (2019). Diretrizes Diagnósticas e Terapêuticas de Neoplasia maligna epitelial de ovário, 419, 109,.

Brasil. Ministério da Saúde. Secretaria de Ciência, Tecnologia e Insumos Estratégicos. Departamento de Gestão e Incorporação de Tecnologias em Saúde. (2016). Entendendo a Incorporação de Tecnologias em Saúde no SUS : como se envolver [recurso eletrônico], . 34 p.

Bristow, R. E., Baldwin, R. L., Yamada, S. D., Korc, M., \& Karlan, B. Y. (1999). Altered expression of transforming growth factor-beta ligands and receptors in primary and recurrent ovarian carcinoma, Cancer ,85 (3), 658-668.

Moura, A.C., de Barros Júnior, A.P., Gouveia, G.C., \& de Oliveira, V.R (2018). Diretrizes Oncológicas: Câncer de Ovário. cap. 23, p.333-352.

Carreno, B. M., Magrini, V., Becker-Hapak, M., Kaabinejadian, S., Hundal, J., Petti, A. A., Ly, A., Lie, W. R., Hildebrand, W. H., Mardis, E. R., \& Linette, G. P. (2015). Cancer immunotherapy. A dendritic cell vaccine increases the breadth and diversity of melanoma neoantigen-specifc T cells. Science, 348(6236), $803-8$.

Cesario, M. S. A., Carneiro, A. M. F.a, \& Dolabela, M. F. (2020). Tratamento medicamentoso em pacientes neonatos com cardiopatia congênita: apontamentos a partir da literatura recente. Research, Society And Development, 9(11). http://dx.doi.org/10.33448/rsd-v9i11.9946

Chen, X. S., Nie, X. Q., Chen, C. M., Wu, J. Y., Wu, J., Lu, J. S., Shao, Z. M., Shen, Z. Z. \& Shen, K. W. (2010). Weekly paclitaxel plus carboplatin is an effective nonanthracycline-containing regimen as neoadjuvant chemotherapy for breast câncer. Annals of Oncology, 21: 961-967. doi:10.1093/annonc/mdq041

Chen, X. S., Nie, X. Q., Chen, C. M., Wu, J. Y., Wu, J., Lu, J. S., Shao, Z. M., Shen, Z. Z., \& Shen, K. W. (2010). Weekly paclitaxel plus carboplatin is an effective nonanthracycline-containing regimen as neoadjuvant chemotherapy for breast câncer. Annals of Oncology 21, 961-967. doi:10.1093/annonc/mdq041.

Colon-Otero, G., Weroha, S. J., Foster, N. R., Haluska, P., Hou, X., Wahner-Hendrickson, A. E., Jatoi, A., Block, M. S., Dinh, T. A., Robertson, M. W., \& Copland, J. A. (2017). Phase 2 trial of everolimus and letrozole in relapsed estrogen receptor-positive high-grade ovarian cancers. Gynecologic Oncology, 146, $64-68$.

Comitê de Consenso (2002). Segundo Consenso de Granada sobre problemas relacionados com medicamentos. Ars Pharm., 43(3-4), 175-184.

Cruz, L. O., Almeida, M. K. C., \& Dolabela, M. F . (2020). Contributions of complementary therapies and other strategies to the treatment of schizophrenia: an integrative review. Research, Society And Development, 9(11). http://dx.doi.org/10.33448/rsd-v9i11.10376

Dale, W., Mohile, S. G., Eldadah, B. A., Trimble, E. L., Schilsky, R. L., Cohen, H. J., Muss, H. B., Schmader, K. E., Ferrell, B., Extermann, M., Nayfield, S. G., \& Hurria, A. (2012). Biological, clinical, and psychosocial correlates at the interface of cancer and aging research. J. Natl. Cancer Inst. 104, 581-589.

del Carmen, M. G., Supko, J. G., Horick, N. K., Rauh-Hain, J. A., Clark, R. M., Campos, S. M., Krasner, C. N., Atkinson, T., \& Birrer, M. J. (2016). Phase I/II Study of Carboplatin and Pralatrexate in Patients with Recurrent Platinum Sensitive Ovarian, Fallopian Tube or Primary Peritoneal Cancer. Cancer, 122: 3297-3306. DOI: 10.1002 / cncr.30196.

del Carmen, M. G., Fuller, A. F., Matulonis, U., Horick, N. K., Goodman, A., Duska, L. R., Penson, R., Campos, S., Roche, M., \& Seiden, M. V. (2003). Phase II trial of anastrozole in women with asymptomatic mullerian câncer. Gynecol. Oncol., 91, 596-602.

Deshpande, P. R., Rajan, S., Lakshmi Sudeepthi, B., \& Abdul Nazir, C. P. (2011). Patient-reported outcomes: a new era in clinical research. Perspect Clin. Res., 2 (4), 137-144.

Du Bois, A., Reuss, A., Harter, P., Pujade-Lauraine, E., Ray-Coquard, I., \& Pfisterer, J. (2010). Potential role of lymphadenectomy in advanced ovarian cancer: a combined exploratory analysis of three prospectively randomized phase III multicenter trials. J Clin Oncol., 28(10),1733-9. doi: 10.1200/JCO.2009.25.3617.

Easton, D. F., Ford, D. \& Bishop, D. T. (1995). Breast and ovarian câncer incidence in BRCA1-mutation carriers. Breast Cancer Linkage Consortium. Am J Hum Genet, 56 (1), 265-71.

Fang, M. Z., Wang, Y., Ai, N., Hou, Z., Sun, Y., Lu, H., Welsh, W., \& Yang, C. S. (2003). Tea polyphenol (-)-epigallocatechin-3-gallate inhibits DNA methyltransferase and reactivates methylation-silenced genes in cancer cell lines. Cancer Res., 63(22), 7563-70.

Gourley, C., Smyth, J. F., Mackean, M., Stevenson, A., Williams, A., Reed, T. R. N., Vasey, P., Gabra, H., \& Langdon, S. (2006). Phase II study of letrozole in estrogen receptor (ER) positive relapsed epithelial ovarian cancer (EOC). J. Clin. Oncol., 24 (18_suppl), 5025.

Graziani, S. R., Vital, C. G., Morikawa1, A. T., Van Eyll, B. M., Junior, H. J. F., Filho, R. K., \& Maranhão, R. C. (2017). Phase II study of paclitaxel associated with lipid core nanoparticles (LDE) as third-line treatment of patients with epithelial ovarian carcinoma. Med Oncol, 34:151. 
Green, M. (2001). A single, fixed-dose of Pegfilgrastin given once-per-chemotherapy cycle is as effective as daily Filgrastrim in the management of neutropenia in high-risk breast cancer. European Journal of Cancer, 37, S146-S147. doi:10.1016/s0959-8049(01)81032-7

Haefele, A., Word, B., Yongmei, X., Hammons, G. J., \& Lyn-Cook, B. D. (2007). Indole-3-carbinol (I3C) modulates expression of DNA methyltransferases 1, $3 \mathrm{a}$, and $3 \mathrm{~b}$ in pancreatic cancer cells: effects of gender and a novel $(\mathrm{C} \rightarrow \mathrm{T})$ polymorphism in the promoter region of DNMT 3b. Int J Cancer Prev., 2(4), 24555 .

Hurria, A., Cirrincione, C. T., Muss, H. B., Kornblith, A. B. Barry, W., Artz, A. S., Schmieder, L., Ansari, R., Tew, W. P., Weckstein, D., Kirshner, J., Togawa, K., Hansen, K., Katheria, V., Stone, R., Galinsky, I., Postiglione, J., \& Cohen, H. J. (2011). Implementing a geriatris assessment in cooperative group clinical cancer trials: CALGB 360401, J. Clin. Oncol., 29, 1290-1296.

Hurria, A., Dale, W., Mooney, M., Rowland, J. H., Ballman, K. V., Cohen, H. J., Muss, H. B., Schilsky, R. L., Ferrell, B., Extermann, M., Schmader, K. E., \& Mohile, S. G. (2014). Designing therapeutic clinical trials for older and frail adults with cancer: U13 conference recommendations. J. Clin. Oncol., 32, 25872594.

Hurria, A., Levit, L. A., Dale, W, Mohile, S. G. Muss, H. B., Fehrenbacher, L., Lichtman, S. M., Bruinooge, S. S., Peres-de-Celis, E., Tew, W. P., Postow, M. A., \& Cohen, H. J. (2015). Improving the evidence base for treating older adults with cancer: American Society of Clinical Oncology Statement. J. Clin. Oncol., 33, 3826-3833.

Hurria, A., Naylor, M., \& Cohen, H. J. (2013). Improving the quality of cancer care in an aging population: recommendations from an IOM report, JAMA, 310, 1795-1796.

Jiang, X-P., Rui, X-H., Guo, C-X., Huang, Y-Q., Li, Q. \& Xu, Y. (2017). A network meta-analysis of eight chemotherapy regimens for treatment of advanced ovarian cancer. Oncotarget.,8 (12), 19125-36.

Kandalaft, L. E., Powell Jr., D. J., Singh, N., \& Coukos, G. (2011). Immunotherapy for ovarian cancer: what's next? J. Clin. Oncol. 29 (7), $925-933$.

Kavanagh, J. J., Hu, W., Fu, S., Deavers, M., Moore, C., Coleman, R. L., Levenback, C. F., Shen, D., Zheng, G., Yf, L., Mueller, P., Gershenson, D. M.,

Zheng, H., \& Li, Y. F. (2007). Anti-tumor activity of letrozole in patients with recurrent advanced low malignant potential or low-grade serous ovarian tumors. J. Clin. Oncol., 25 (18_suppl), 5582.

Kiselev, V. I., Ashrafyan, L. A., Muyzhnek, E. L., Gerfanova, E. V., Antonova, I. B., Aleshikova, O. I., \& Sarkar, F. H. (2018). A new promising way of maintenance therapy in advanced ovarian cancer: a comparative clinical study. BMC Cancer, 18:904.

Komiyama, S., Kugimiya, T., \& Kubushiro, K. (2018). Safety and efficacy of neoadjuvant chemotherapy containing bevacizumab and interval debulking surgery for advanced epithelial ovarian cancer: A feasibility study. J. Surg. Oncol., 118, 687-693. DOI: 10.1002/jso.25187.

Krasner, C. N., Debernardo, R. L., Findley, M., Penson, R., Matulonis, U., Atkinson, T., Roche, M., \& Seiden, M. V. (2005). Phase II trial of anastrazole in combination with gefitinib in women with asymptomatic mullerian câncer. J. Clin. Oncol., 23 (16_suppl), 5063.

Lawrie, T. A., Winter-Roach, B. A., Heus. P. \& Kitchener, H. C. (2015). Adjuvant (post-surgery) chemotherapy for early stage epithelial ovarian cancer. Cochrane Database Syst Rev., 17 (12). https://doi.org/10.1002/14651858.CD004706.pub5.

Li, Y., Kong, D., Wang, Z., \& Sarkar, F. H. (2010) Regulation of microRNAs by natural agents: an emerging field in chemoprevention and chemotherapy research. Pharm Res., 27(6), 1027-41.

Li, Y., Li, X., \& Guo, B. (2010). Chemopreventive agent 3,3'-diindolylmethane selectively induces proteasomal degradation of class I histone deacetylases. Cancer Res., 70(2), 646-54.

Li, Y., \& Tollefsbol, T. O. (2010). Impact on DNA methylation in cancer prevention and therapy by bioactive dietary components. Curr Med Chem., 17(20), 2141-51.

Li, Y. F., Hu, W., Fu, S. Q., Li, J. D., Liu, J. H., \& Kavanagh, J. J. (2008). Aromatase inhibitors in ovarian cancer: is there a role? Int. J. Gynecol. Cancer ,18, $600-614$.

Li, F., Deng, L., Jackson, K. R., Talukder, A. H., Katailiha, A. S., Bradley, S. D., Zou, Q., Chen, C., Huo, C., Chiu, Y., Stair, M., Feng, W., Bagaev, A., Kotlov, N., Svekolkin, V., Ataullakhanov, R., Miheecheva, N., Frenkel, F., Wang, Y., Zhang, M., Decker, W. K., Sonnemann, H. M., Roski, J., Forget, M. E., Davies, M. A., Bernatchez, C., Yee, C., Basset, R., Hwu, P., Du, X., \& Lizee, G. (2021) Neoantigen vaccination induces clinical and immunologic responses in non-small cell lung cancer patients harboring EGFR mutations. J Immunother Cancer, 9(7), e002531. doi: 10.1136/jitc-2021-002531.

Luiz, B. M., Miranda, P. F., Maia, E. M. C., Machado, R. B., Giatti, M. J. L., Antico Filho, A., \& Borges, J. B. R. (2009) Estudo Epidemiológico de Pacientes com Tumor de Ovário no Município de Jundiaí no Período de Junho de 2001 a Junho de 2006. Revista Brasileira de Cancerologia, 55(3), $247-253$.

Lyn-Cook, B. D., Mohammed, S. I., Davis, C., Word, B., Haefele, A., Wang, H., \& Hammons, G. (2010). Gender differences in gemcitabine (Gemzar) efficacy in cancer cells: effect of indole-3-carbinol. Anticancer Res., 30(12), 4907-13.

Maier-Lenz, H., Hauns, B., Haering, B., Koetting, J., Mross, K., Unger, C., Bauknecht, T., du Bois, A., Meerpohl, H. G., Hollaender, N., \& Diergarten, K. (1997). Phase I study of paclitaxel administered as a 1-hour infusion: toxicity and pharmacokinetics. Semin Oncol., 24(6 Suppl 19),S19-16-S19-1.

Moura, A. C. C. de, Barros Júnior, A. P. de, Gouveia, G. de C \& Oliveira, V. R. (2018). Câncer De Ovário. Diretrizes Oncológicas, 23, 333-352.

Oh, J., Barve, M., Matthews, C. M., Koon, E. C., Heffernan, T. P., Fine, B., Grosen, A., Bergman, M. K., Fleming, E. L., DeMars, L. R., West, L., Spitz, D. L., Goodman, H., Hancock, K. C., Wallraven, G., Kumar, P., Bognar, E., Manning, L., Pappen, B. O., Adams, N., Senzer, N., \& Nemunaitis, J. (2016). Phase II study of Vigil ${ }^{\circ}$ DNA engineered immunotherapy as maintenance in advanced stage ovarian câncer. Gynecologic Oncology, 143, 504-510 
Osborne, C. K., Shou, J., Massarweh, S. \& Schiff, R. (2005). Crosstalk between estrogen receptor and growth factor receptor pathways as a cause for endocrine therapy resistance in breast câncer. Clin. Cancer Res., 11, 865s-870s.

Ott, P. A., Govindan, R., Naing, A., Friedlander, T. W., Margolin, K., Lin, J. J., Bhardawaj, N., Hellman, M. D., Srinivasan, L., Greshock, J., Moles, M., Gaynor, R. B., Goldstein, M. J., \& Hu-Lieskovan, S. (2018). A personal neoantigen vaccine, NEO-PV-01, with anti-PD1 induces broad de novo anti-tumor immunity in patients with metastatic melanoma, NSCLC and bladder cancer. Ann Oncol., 29(suppl_8), viii400-41. https ://doi.org/10.1093/annonc/mdy288.

Ott, P. A., Hu, Z., Keskin, D. B., Shukla, S. A., Sun, J., Bozym, D. J., Zhang, W., Luoma, A., Giobbie-Hurder, A., Peter, L., Chen, C., Olive1, O., Carter, T. A., Li, S., Lieb, D. J., Eisenhaure, T., Gjini, E., Stevens, J., Lane, W. J., Javeri, I., Nellaiappan, K., Salazar, A. M., Daley, H., Seaman, M., Buchbinder, E. I., Yoon, C. H., Harden, M., Lennon, N., Gabriel, S., Rodigo, S. J., Barouch, D. H., Aster, C. J., Getz, G., Wucherpfennig, K., Neuberg, D., Ritz, J., Lander, E. S., Fritsch, E. F., Hacohen, N., \& Wu1, C. J. (2017). An immuno- genic personal neoantigen vaccine for patients with melanoma. Nature, 547, $217-21$.

Pandey, M., Shukla, S., \& Gupta, S. (2010). Promoter demethylation and chromatin remodeling by green tea polyphenols leads to re-expression of GSTP1 in human prostate cancer cells. Int J Cancer, 126(11), 2520-33.

Papadimitriou, C. A., Markaki, S., Siapkaras, J., Vlachos, G., Efstathiou, E., Grimani, I., Hamilos, G., Zorzou, M., \& Dimopoulos, M., (2004). Hormonal therapy with letrozole for relapsed epithelial ovarian cancer. Long-term results of a phase II study. Oncology, 66, $112-117$.

Raber-Durlacher, J. E., Weijl, N. I., Abu Saris, M., de Koning, B., Zwinderman, A. H \& Osanto, S.(2000). Oral mucositis in patients treated with chemotherapy for solid tumors: a retrospective analysis of 150 cases. Support Care Cancer, 8:366-71.

Rauh-Hain, J. A., Melamed, A., Wright, A., Gockley, A., Clemmer, J. T., Schorge, J. O., Del Carmen, M. G., \& Keating, N. L. (2017). Overall Survival Following Neoadjuvant Chemotherapy vs Primary Cytoreductive Surgery in Women With Epithelial Ovarian Cancer: Analysis of the National Cancer Database. JAMA Oncol., 3 (1),76-82.

Sahin, U., Derhovanessian, E., Miller, M., Kloke, B. P., Simon, P., Lower, M., Bukur, V., Tadmor, A. D., Luxemburger, U., Schrörs, B., Omokoko, T., Vormehr, M., Albrecht, C., Paruzynski, A., Kuhn, A.N., Buck, J., Heesch, S., Schreeb, K.H., Müller, F., ..., Türeci, O. (2017). Personalized RNA mutanome vaccines mobilize poly-specifc therapeutic immunity against cancer. Nature, 547(7662), 222-6.

Sarivalasis, A., Boudousquié. C., Balint, K., Stevenson, B. J., Gannon, P. O., Iancu, E. M., Rossier, L., Lluesma, S. M., Mathevet, P., Sempoux, C., Coukos, G., Dafni, U., Harari, A., Bassani-Sternberg, M., \& Kandalaft, L. E. (2019). A Phase I/II trial comparing autologous dendritic cell vaccine pulsed either with personalized peptides (PEP-DC) or with tumor lysate (OC-DC) in patients with advanced high-grade ovarian serous carcinoma. Transl Med, 17:391.

Schietinger, A., Philip, M., \& Schreiber, H. (2008). Specifcity in cancer immunotherapy. Semin Immunol., 20(5), $276-85$.

Senzer, N., Barve, M., Kuhn, J., Melnyk, A., Beitsch, P., Lazar, M., Lifshitz, S., Magee, M., Oh, J., Mill, S.W., Bedell, C., Higgs, C., Kumar, P., Yu, Y., Norvell, F., Phalon, C., Taquet, N., Rao, D.D., Wang, Z., ... Nemunaitis, J. (2012). Phase I trial of "bishRNAi(furin)/GMCSF DNA/autologous tumor cell" vaccine (FANG) in advanced câncer. Mol. Ther. 20 (3), 679-686.

Senzer, N., Barve, M., Nemunaitis, J., Kuhn, J., Melnyk, A., Beitsch, P., Magee, M., Oh, J., Bedell, C., Kumar, P., Rao, D.D., Pappen, B.O., Wallraven, G., Brunicardi, F.C., \& Maples, P.B. (2013). Long term follow up: phase I trial of "bi-shRNA furin/GMCSF DNA/Autologous Tumor Cell" immunotherapy (FANG ${ }^{\mathrm{TM}}$ ) in advanced câncer. Journal of Vaccines and Vaccination, 4 (8), 209.

Simoncini, T., Hafezi-Moghadam, A., Brazil, D. P., Ley, K., Chin, W. W., \& Liao, J. K. (2000). Interaction of oestrogen receptor with the regulatory subunit of phosphatidylinositol-3- OH kinase. Nature, 407, 538-541

Sopik, V., Rosen, B., Giannakeas, V., \& Narod, S. A. (2015). Why have ovarian cancer mortality rates declined? Part III. Prospects for the future. Gynecol Oncol., 138, 757-61.

Strickland, D. K., Kounnas, M. Z., \& Argraves, W. S. (1995). LDL receptor related protein: a multiligand receptor for lipoprotein and proteinase catabolism. FASEB J., 9:890-8

Struewing, J. P., Hartge, P., Wacholder, S., Baker, S. M., Berlin,M., McAdams, M., Timmerman, M. M., Brody, L. C., \& Tucker, M. A. (1997). The risk of cancer associated with specific mutations of BRCA1 and BRCA2 among Ashkenazi Jews. N Engl J Med, 336 (20), 1401-8. 10.1056/NEJM199705153362001

Tchekmedyian, N. S., Liem, A. K., Quan, E. T., Burtzo, D. M. \& Ucar, K. (2006). Aromatase inhibitor therapy for estrogen receptor positive ovarian câncer. J. Clin. Oncol., 24 (18_suppl), 15038.

Verma, S., Alhayki, M., Le, T., Baines, K., Rambout, L., Hopkins, L. \& Fung Kee Fung, M. (2006). Phase II study of exemestane (E) in refractory ovarian cancer (ROC). J. Clin. Oncol., 24 (18_suppl), 5026.

Von Gruenigen, V. E., Huang, H. Q., Beumer, J. H., Lankes, H. A., Tew, W., Herzog, T., Hurria, A., Mannel, R. S., Rizack, T., Landrum, L. M., Rose, P. G., Salani, R., Bradley, W. H., Rutherford, T. J., Higgins, R. V., Secord, A. A., \& Fleming, G. (2017). Chemotherapy completion in elderly women with ovarian, primary peritoneal or fallopian tube cancer - An NRG oncology/Gynecologic Oncology Group study. Gynecologic Oncology, $144,459-467$.

Weiss, R.B., Donehower, R. C., Wiernik, P. H., Ohnuma, T., Gralla, R. J., Trump, D. L., Baker Jr, J. R., Van Echo, D. A., Von Hoff, D. D., \& Leyland-Jones, B. (1990) Hypersensitivity reactions from Taxol. J Clin Oncol., 8, 1263-8.

Wildiers, H., Heeren, P., Puts, M., Topinkova, E., Janssen-Heijnen, M. L., Exterman, M., Falandry, C., Artz, A., Brain, E., Colloca, G., Flamaing, J., Karnakis, D., Kenis, C., Audidio, R. A., Mohile, S., Repetto, L., Leeuwen, B. V., Milisen, K., \& Hurria, A. (2014). International Society of Geriatric Oncology consensus on geriatric assessment in older patients with câncer. J. Clin. Oncol., 32 (24), 2595-2603.

World Ovarian Cancer Coalition. About ovarian câncer. (2021). https://worldovariancancercoalition.org/about-ovarian-cancer/what-is-ovarian-cancer/. 
Research, Society and Development, v. 10, n. 16, e497101620638, 2021

(CC BY 4.0) | ISSN 2525-3409 | DOI: http://dx.doi.org/10.33448/rsd-v10i16.20638

Wright, A. A., Bohlke, K., Armstrong, D. K., Bookman, M. A., Cliby, W. A., Coleman, R. L., Dizon, D. S., Kash, J. J., Meyer, L. A., Moore, K. N., Olawaiye, A. B., Oldham, J., Salani, R., Sparaccio, D., Tew, W. P., Vergote, I., \& Edelson, M. I. (2016). Neoadjuvant Chemotherapy for Newly Diagnosed, Advanced Ovarian Cancer: Society of Gynecologic Oncology and American Society of Clinical Oncology Clinical Practice Guideline. J Clin Oncol Off J Am Soc Clin Oncol., 34(28), 3460-73.

Wu, T. Y., Khor, T. O., Su, Z. Y., Saw, C. L., Shu, L., Cheung, K. L., Huang, Y., Yu, S., \& Kong, A. T. (2013). Epigenetic modifications of Nrf2 by 3,3'diindolylmethane in vitro in TRAMP C1 cell line and in vivo TRAMP prostate tumors. The AAPS J., 15(3):864-74. 